\title{
Saturation effects in low-x DIS structure functions and related hadronic total cross sections
}

\author{
Francesco Giovanni Celiberto ${ }^{1,2, \star}$, Laszlo Jenkovszky ${ }^{3, \star \star}$, and Volodymyr Myronenko $0^{4, \star \star \star}$ \\ ${ }^{1}$ Dipartimento di Fisica, Università della Calabria, Arcavacata di Rende, 87036 Cosenza, Italy \\ ${ }^{2}$ Istituto Nazionale di Fisica Nucleare, Gruppo Collegato di Cosenza, \\ Arcavacata di Rende, 87036 Cosenza, Italy \\ ${ }^{3}$ Bogolyubov Institute for Theoretical Physics (BITP), Ukrainian National Academy of Sciences 14-b, \\ Metrologicheskaya str., Kiev, 03680, Ukraine \\ ${ }^{4}$ Deutsches Elektronen-Synchrotron DESY, Hamburg, Germany
}

\begin{abstract}
.
High-energy nucleon total cross sections are related to low-x DIS structure functions by using the additive quark model.
\end{abstract}

In the additive quark model, the hadron-hadron total cross section can be written as a product of the cross sections of the constituents, $\sigma_{q q}[1,2]$, e.g.

$$
\sigma(s)_{p p}^{t}=\sigma_{q q}\left[n_{V}+n_{S}(s)\right]^{2},
$$

where $n_{V}$ is the number of valence quarks and $n_{S}(s)$ is that of sea quarks, their number increasing with energy.

It was suggested in Refs. [1,2] that the increasing number of sea quarks is related to the Bjorken scaling-violating contribution to the deep inelastic lepton-hadron structure function (DIS SF), namely to the momentum fraction of the relevant quarks given by the integral over the DIS structure function $F_{2}\left(x, Q^{2}\right)$. In Ref. [1] a simple model for the DIS structure function, known at those times, was used, resulting in the following expression for the total cross section, compatible with the data

$$
\sigma(s)_{p p}^{t}=\sigma_{q q} n_{V}^{2}\left(1+0.0 .16 \ln \left(s / Q_{0}^{2}\right)\right),
$$

where $\sigma_{q q}$ is a free parameter, $Q_{0}^{2}$ was fitted to the DIS data, and $n_{V}=3$.

In Ref. [2] the DIS SF was related to hadronic cross sections by means of finite-energy sum rules in $Q^{2}$. $[3,4]$.

The number of quarks in a reaction can be calculated from the SF by means of sum rules. see e.g.

In Ref. [5] following ansatz for the small- $x$ singlet part (labelled by the upper index $S, 0$ ) of the proton structure function, interpolating between the soft (VMD, Pomeron) and hard (GLAP evolution) regimes was proposed:

\footnotetext{
^e-mail: francescogiovanni.celiberto@fis.unical.it

$\star \star$ e-mail: jenk@bitp.kiev.ua

$\star \star \star$ e-mail: volodymyr.myronenko@desy.de
} 

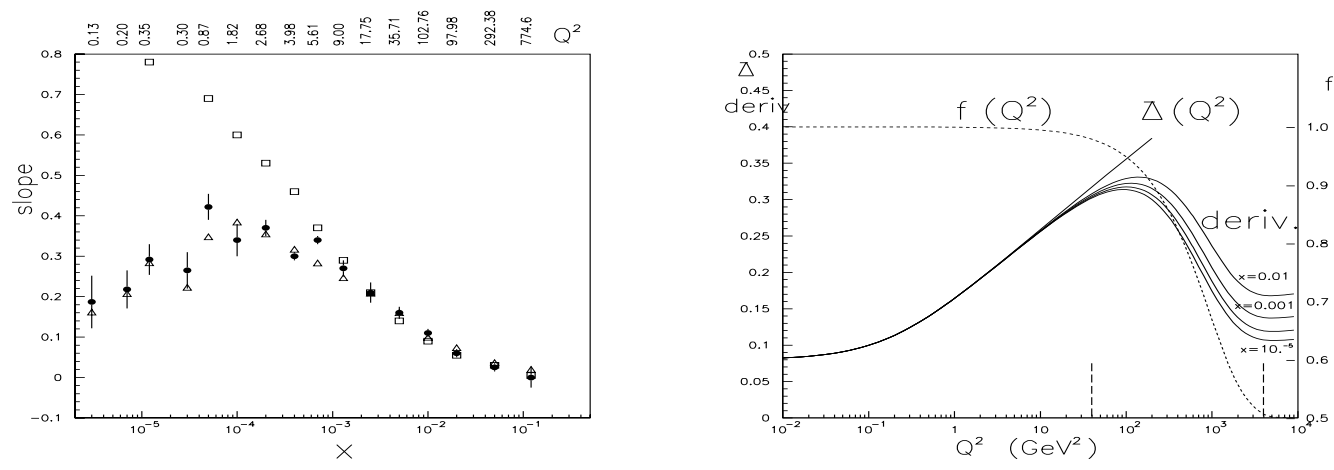

Figure 1. Slope of the structure function $F_{2}\left(x, Q^{2}\right)$.

$$
F_{2}^{(S, 0)}\left(x, Q^{2}\right)=A\left(\frac{Q^{2}}{Q^{2}+a}\right)^{1+\widetilde{\Delta}\left(Q^{2}\right)} e^{\Delta\left(x, Q^{2}\right)},
$$

with the "effective power"

$$
\widetilde{\Delta}\left(Q^{2}\right)=\epsilon+\gamma_{1} \ln \left(1+\gamma_{2} \ln \left[1+\frac{Q^{2}}{Q_{0}^{2}}\right]\right),
$$

and

$$
\Delta\left(x, Q^{2}\right)=\left(\widetilde{\Delta}\left(Q^{2}\right) \ln \frac{x_{0}}{x}\right)^{f\left(Q^{2}\right)},
$$

where

$$
f\left(Q^{2}\right)=\frac{1}{2}\left(1+e^{-Q^{2} / Q_{1}^{2}}\right)
$$

At small and moderate values of $Q^{2}$, the exponent $\widetilde{\Delta}\left(Q^{2}\right)$ (3.2) may be interpreted as a $Q^{2}$ dependent "effective Pomeron intercept", as shown in Fig. 1.

The function $f\left(Q^{2}\right)$ has been introduced in order to provide for the transition from the Regge behaviour, where $f\left(Q^{2}\right)=1$, to the asymptotic solution of the GLAP evolution equation, where $f\left(Q^{2}\right)=1 / 2$.

In Ref. [5] the above singlet SF was appended by a non-singlet part, important at large values of $x$. The parameters were fitted to the DIS data in a wide range of $x$ and $Q^{2}$. The values of the fitted parameters are: $A=0.1623, a=0.2916 \mathrm{GeV}^{2}, \quad \gamma_{2}=0.01936, Q_{0}^{2}=0.1887 \mathrm{GeV}^{2}, Q_{1}^{2}=916.1$ $\mathrm{GeV}^{2} ; x_{0}=1, \epsilon=0.08, \quad \gamma_{1}+2.4$ were fixed (by QCD-related arguments). The resulting fits and more details can be found in Ref. [5].

The proton-proton total cross section is cast by integrating Eqs. (3) between $x=0$ and $x=1$. At high energies, only the singlet part of the SF, Eqs. (3) (the "Pomeron") is relevant. Integration can be 

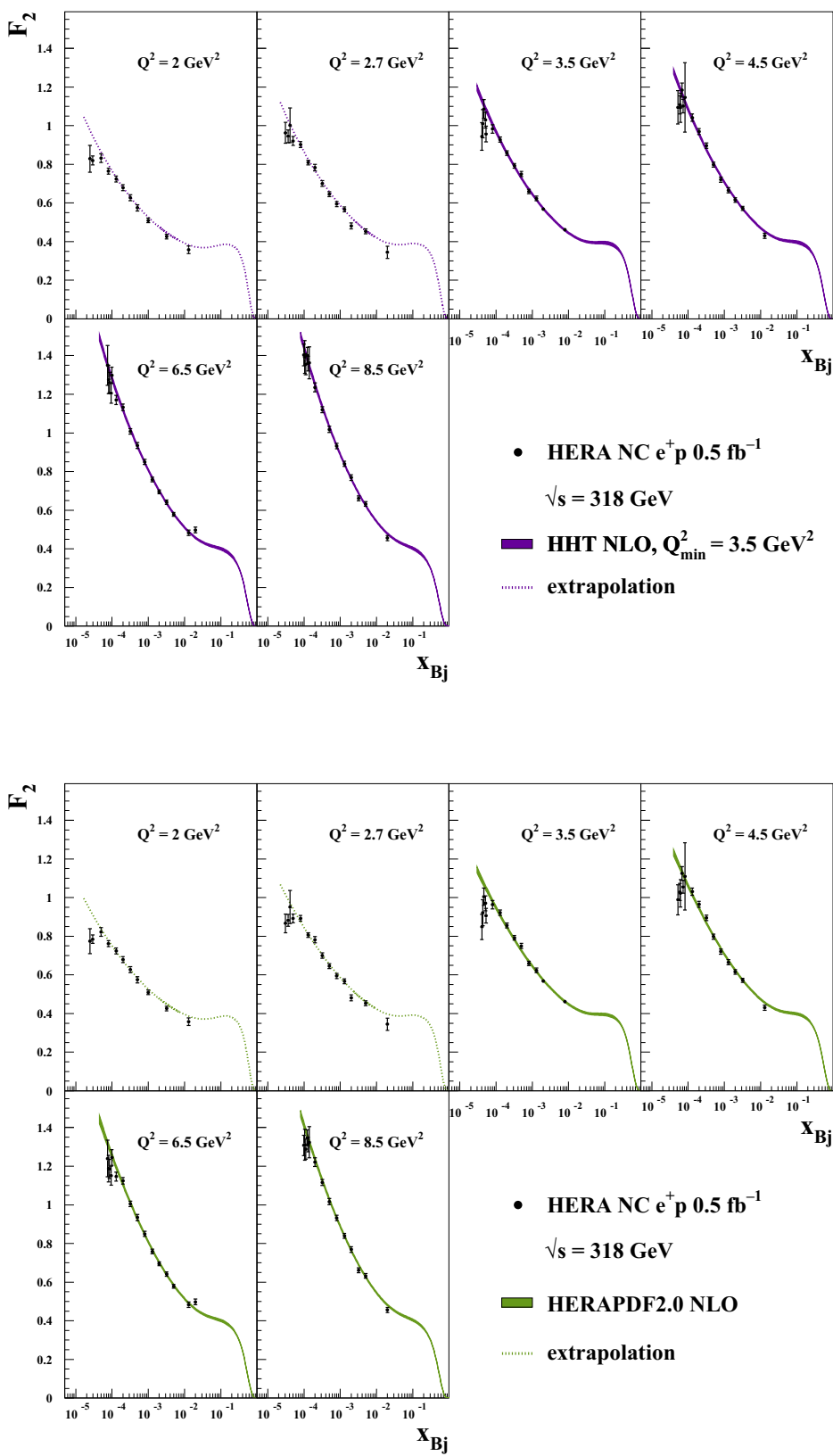

Figure 2. HHT (top) and HERAPDF2.0 (bottom) NLO predictions of the $F_{2}$ structure function at $Q_{\min }^{2}=3.5 \mathrm{GeV}^{2}$, compared to extracted values. For more details, see Ref. [7]. 
performed numerically. The result is in reasonable agreement with the data on $p p$ total cross sections, including those from the LHC.

The operator-product expansion beyond leading twist has diagrams in which two, three or four gluons may be exchanged in the $t$-channel such that these gluons may be viewed as recombining. This recombination could lead to gluon saturation. The colour-dipole framework also inspired a phenomenological model of saturation by Golec-Biernat-Wüsthoff (GBW), in which the onset of saturation is characterised as the transition from a "soft" to a "hard" scattering regime. This occurs along a "critical line" in the $x_{B j}, Q^{2}$ plane.

Recently new results on low- $x$ DIS parton distrubutions (PDF and SF) have appeared [6, 7]; they show an intriguing change of regime towards smallest values of $x$ (Fig. 2) - possible saturation effect?

We intend to investigate its impact on the asymptotic behaviour of the total cross sections.

\section{Acknowledgement}

L.J. thanks the Organizers of the Quarks2016 conference for their warm hospitality and support.

\section{References}

[1] L.L. Jenkovszky and B.V. Struminsky: Violation of scale invariance in deep-inelastic leptonhadron scattering and rise of hadronic cross-sections, ITP-77-37E Preprint, Kiev, 1977.

[2] L.L. Jenkovszky and B.V. Struminsky: Yadernaya Fizika (English translation: Sov. J. Nucl. Phys.), 38(1983), 1568; see also: http://inspirehep.net/record/1456006/files/ $m p i_{a} t_{l} h c_{2} 015 . p d f ;$ http://indico.ictp.it/event/a14280/, p.171.

[3] F.E. Close, An introduction to quarks and partons, Academic Press Inc. (London) Ltd., 1979.

[4] R.G. Roberts, The structure of the proton, Cambridge University Press, 1990.

[5] P. Desgrolard, Laszlo L. Jenkovszky, F. Paccanoni, Interpolating between soft and hard dynamics in deep inelastic scattering. LYCEN-9808, Eur. Phys. J. C7 (1999) 263-270; hep$\mathrm{ph} / 9803286$.

[6] H. Abramowicz et al. [H1 and ZEUS Collaborations], Combination of measurements of inclusive deep inelastic $e^{ \pm} p$ scattering cross sections and QCD analysis of HERA data, Eur. Phys. J. C 75 (2015) no.12, 580 doi:10.1140/epjc/s10052-015-3710-4 [arXiv:1506.06042 [hep-ex]].

[7] I. Abt, A. M. Cooper-Sarkar, B. Foster, V. Myronenko, K. Wichmann and M. Wing, Study of HERA ep Data at Low $Q^{2}$ and Low $x_{B_{j}}$ and the Need for Higher-Twist Corrections to Standard pQCD Evolution, arXiv:1604.02299 [hep-ph]. 